\title{
100G Flexible IM-DD 850 nm VCSEL Transceiver with Fractional Bit Rate Using Eight- Dimensional PAM
}

Lu, Xiaofeng; Lyubopytov, Vladimir; Chorchos, ukasz; Stepniak, Grzegorz ; Agustin, Mikel; Kropp, Jorg R.; Ledentsov, Nikolay; Shchukin, Vitaly A.; Ledentsov, Nikolay; Turkiewicz, Jarosaw P.

Total number of authors:

11

Published in:

2017 European Conference on Optical Communication (ECOC)

Link to article, DOI:

10.1109/ECOC.2017.8346136

Publication date:

2017

Document Version

Peer reviewed version

Link back to DTU Orbit

Citation (APA):

Lu, X., Lyubopytov, V., Chorchos, ., Stepniak, G., Agustin, M., Kropp, J. R., Ledentsov, N., Shchukin, V. A., Ledentsov, N., Turkiewicz, J. P., \& Tafur Monroy, I. (2017). 100G Flexible IM-DD 850 nm VCSEL Transceiver with Fractional Bit Rate Using Eight-Dimensional PAM. In 2017 European Conference on Optical Communication (ECOC) IEEE. https://doi.org/10.1109/ECOC.2017.8346136

\section{General rights}

Copyright and moral rights for the publications made accessible in the public portal are retained by the authors and/or other copyright owners and it is a condition of accessing publications that users recognise and abide by the legal requirements associated with these rights.

- Users may download and print one copy of any publication from the public portal for the purpose of private study or research.

- You may not further distribute the material or use it for any profit-making activity or commercial gain

- You may freely distribute the URL identifying the publication in the public portal 


\title{
100G Flexible IM-DD 850 nm VCSEL Transceiver with Fractional Bit Rate Using Eight-Dimensional PAM
}

\author{
Xiaofeng Lu ${ }^{(1)}$, Vladimir S. Lyubopytov ${ }^{(1)}$, Łukasz Chorchos ${ }^{(2)}$, Grzegorz Stepniak ${ }^{(2)}$, Mikel Agustin ${ }^{(3)}$, \\ Jörg-R. Kropp ${ }^{(3)}$, Nikolay N. Ledentsov ${ }^{(3)}$, Vitaly A. Shchuki( ${ }^{(3)}$, Nikolay Ledentsov Jr. ${ }^{(3)}$, \\ Jarosław P. Turkiewicz ${ }^{(2)}$, Idelfonso Tafur Monroy ${ }^{(1)}$ \\ (1) DTU Fotonik, Technical University of Denmark, 2800 Kgs. Lyngby, Denmark, xilu@fotonik.dtu.dk \\ (2) Institute of Telecommunications, Warsaw University of Technology, 00-661 Warsaw, Poland \\ (3) VI-Systems GmbH, 10623 Berlin, Germany
}

\begin{abstract}
We demonstrate a novel optical transceiver scheme with a net flexible bit rate up to 100Gbit/s with 5 Gbit/s granularity, using an eight-dimensional modulation format family, and investigate its performance on capacity, reach, and power tolerance.
\end{abstract}

\section{Introduction}

Optimizing the utilization of hardware resources and achieving a target transmission performance is a fundamental objective in the design of intensity-modulated direct-detection (IM-DD) transceivers using pulse-amplitude modulation (PAM). As illustration, for a given link configuration, using PAM-4 may underuse available output power; whereas stepping up to PAM-8 can be hindered by insufficient power budget. A solution to this gap is found in the use of multi-dimensional (MD) modulation formats ${ }^{1,2}$, carrying a fractional number of bits per symbol (bit/sym). Furthermore, such MD formats enable bit-rate flexible transmission. The concept of using four-dimensional (4D) formats in a rate flexible transceiver $^{3}$ and a multi-rate IM-DD transceiver using 4D formats ${ }^{4}$ have been proposed. Recently, 8D modulation format has been reported for coherent detection ${ }^{5,6}$ and IM-DD systems ${ }^{7}$.

In this paper, we propose, design and experimentally investigate a flexible IM-DD transceiver with software-controlled bit rate, using a newly designed 8D format family, which has a spectrum efficiency granularity of 0.125 bit/sym. Such flexible transceiver effectively utilizes bandwidth and power budget resources and benefits from the hardware simplicity of PAM- $m$ modulators. By using a fractional number of bit/sym, we demonstrate a net 100Gbit/s transmission over $200 \mathrm{~m}$ OM4 MMF and gross 100Gbit/s over $1 \mathrm{~km} \mathrm{SSMF}$, where otherwise both PAM-4 and PAM-8 fail.

\section{$E_{8}$ Flex- $n$ and bitrate flexible transceiver}

For realizing the novel software-controlled rate flexibility, we design an $8 \mathrm{D}$ format family, i.e. $\mathrm{E}_{8}$ Flex- $n$, together with its fast (de)mapping algorithm and simplified decision scheme. Similar to BB8 format ${ }^{7}, E_{8}$ Flex- $n$ is based on an $\mathrm{E}_{8}$ lattice grid and uses eight temporally consecutive symbols to form an $8 \mathrm{D}$ supersymbol (s-sym). Each s-sym carries $n$ bits, namely $n / 8$ bits per conventional symbol, giving a 0.125 bit/sym granularity. The BER sensitivity increases when reducing the constellation scale, where the minimum Euclidean distance is enlarged or the format set gets sparser. Despite the complex $8 \mathrm{D}$ geometry, each transmitted symbol set is equivalent to a conventional PAM symbol with evenly distributed levels. $\mathrm{E}_{8} \mathrm{Flex}-n$ is derived from BB8 and covers a family of $8 D$ formats. For example, $\mathrm{E}_{8} \mathrm{Flex}-15$ is equivalent to the earlier reported $8 \mathrm{D}$ formats ${ }^{5}$ and E8Flex-16 is equivalent to $\mathrm{BB}^{7}$. Borrowing the methods of the latter, we extend the fast (de)mapping algorithm to all $\mathrm{E}_{8} \mathrm{Flex}-n$ from $n=1$ to an arbitrary number, within a uniform modulation framework. The bit mapping of this modulation is further modified to enable the swift and smooth scale alteration. $E_{8}$ Flex- $n$ modulation is bit-stream based without using the look-up table containing the symbol alphabets. Only bit-level operations are required before the PAM- $m$ modulator. The bit stream volume fed to the modulator is simply controlled by the control bits. During the decision phase, we first apply hard decision on each PAM symbol and make final decision among 2, 4, 8 or 16 candidate 8D points, according to the format scale $(n)$. The proposed scheme uses a fixed baud rate and shifts the bit rate only by using the formats of different scales.

\section{Experimental setup}

For comparison purposes, in the experimental setup (Fig. 1) we use different types of $850 \mathrm{~nm}$ vertical-cavity surface-emitting lasers (VCSELs) a multi-mode (MM) VCSEL, and two single-mode (SM) VCSELs coupled with multi-mode fiber (MMF) and single-mode fiber (SMF) pigtails, respectively. The $-3 \mathrm{~dB}$ bandwidth of the MMVCSEL and SM-VCSELs reaches $18 \mathrm{GHz}$ and $20 \mathrm{GHz}$ respectively, and the optical output power levels reach $4 \mathrm{dBm}$ (MM-VCSEL), 0.5 $\mathrm{dBm}$ (MMF-coupled SM-VCSEL) and $-1.7 \mathrm{dBm}$ (SMF-coupled SM-VCSEL). 
(a)

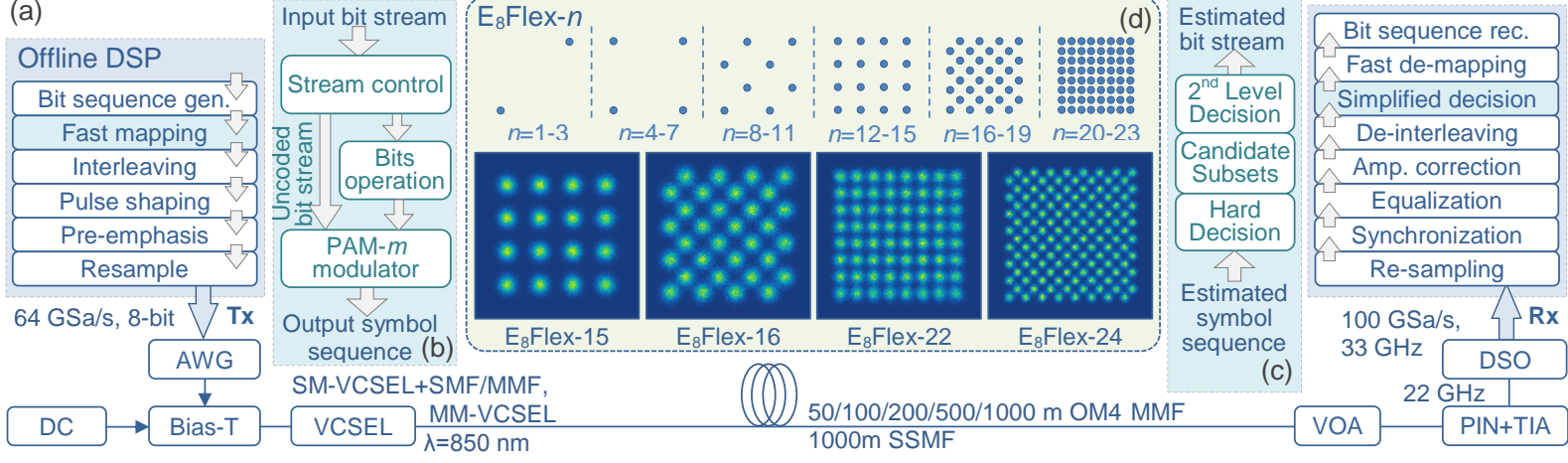

Fig. 1: (a) Scheme of the experimental setup; (b) Scheme of the fast mapping; (c) Scheme of the simplified decision; (d) 2D projections of E8Flex-n constellations.

After transmission through the fiber links of different lengths, the optical signal is detected by a calibrated photoreceiver module with copackaged transimpedance amplifier (TIA). At the transmitter, for each measured trace, 30 Mbit input streams were generated randomly for avoiding the pattern dependent effects and mapped with the fast $E_{8}$ Flex mapper. After interleaving, the symbol sequences were resampled to the desired baud rate and shaped into raised cosine/root raised cosine pulses. The spectra of output sequences were preemphasized, for compensating the electrical spectral roll-off of the transmitter. During the offline digital signal processing (DSP) at the receiver side, the electrical signal was resampled into two times of the baud rate, and after sequence synchronization it was equalized by a T/2 fractional FFE together with an amplitude correction. After de-interleaving, the output bit streams were obtained by the $\mathrm{E}_{8} \mathrm{Flex}$ de-mapper.

\section{Experimental results}

Bandwidth requirement: Fig. 2(a) shows the optical back-to-back (OBTB) performance for different numbers of bit/sym with various baud rates. The red dot curve indicates the gross 108G threshold. The parameters chosen from the shadowed area enable the net 100G
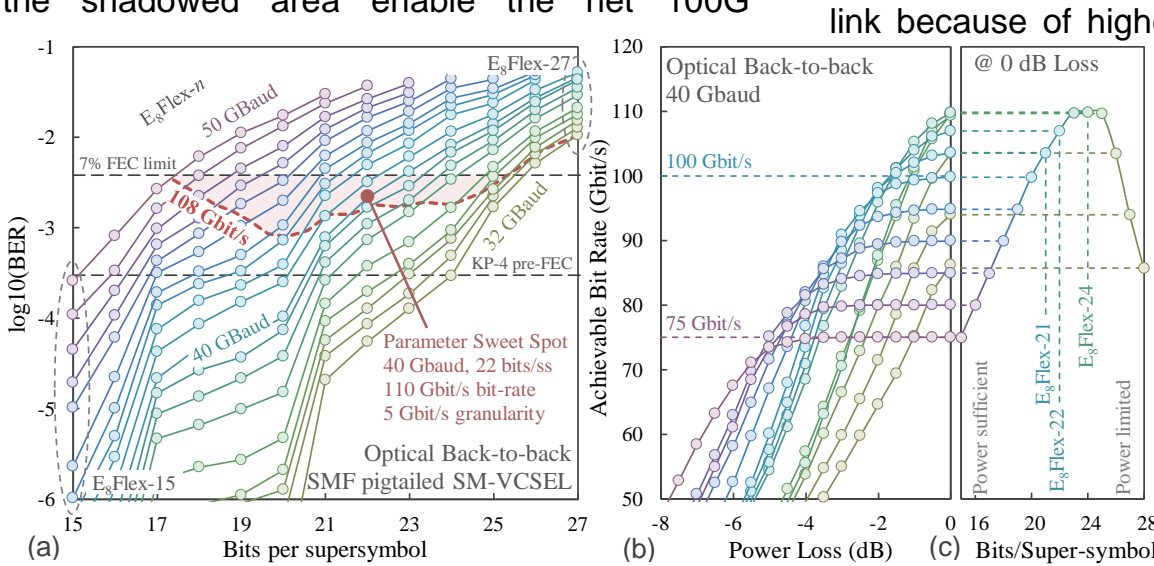

transmission and beyond. It follows that $22 \mathrm{bit} / \mathrm{s}$ sym in 40 Gbaud systems is a fair choice, which gives a trade-off between bit rate and system bandwidth, providing a gross rate of $110 \mathrm{Gbit} / \mathrm{s}$. Thus, in the later tests, we use 40 Gbaud for the system investigation and 32 Gbaud as a reference, which correspond to $5 \mathrm{Gbit} / \mathrm{s}$ and 4 Gbit/s granularity respectively.

Capacity: The OBTB performance of SM-VCSEL shows a transmission potential up to $110 \mathrm{Gbit} / \mathrm{s}$ within the power budget of our experimental setup, see Fig. 2(b). As Fig. 2(c) shows, achievable bit rate starts to saturate at $23 \mathrm{bit} / \mathrm{s}$ sym due to limited power. It implies that $\mathrm{E}_{8} \mathrm{Flex}-22$ has a near optimal utilization of the laser output power and guarantees the net 100G transmission. Another interesting point is $\mathrm{E}_{8} \mathrm{Flex}-21$, which has a lower bit rate but simplest decision procedure among $\mathrm{E}_{8}$ Flex-16 to $\mathrm{E}_{8}$ Flex-23.

VCSEL comparison: Fig. 2(d) shows the system performance in OBTB mode with three different types of VCSELs, demonstrating better behavior of SM-VCSELs compared to MM-VCSEL. Fig. 2(e) provides comparison of SMF- and MMFpigtailed SM-VCSELs for different transmission lengths, showing that MMF-pigtailed SMVCSEL provides slightly better BER performance in case of OBTB and 100m MMF link because of higher output power, but SMFFig. 2: (a) BER performance vs. the number of bits per super-symbol for different symbol rate values; (b,c) Achievable bit
rate for different numbers of bits per super-symbol; (d) BER performance vs. channel capacity for different types of VCSEL modules in OBTB mode; (e) BER difference between MMF- and SMF-pigtailed SM-VCSELs vs. channel capacity for different MMF links at 40 Gbaud symbol rate. 

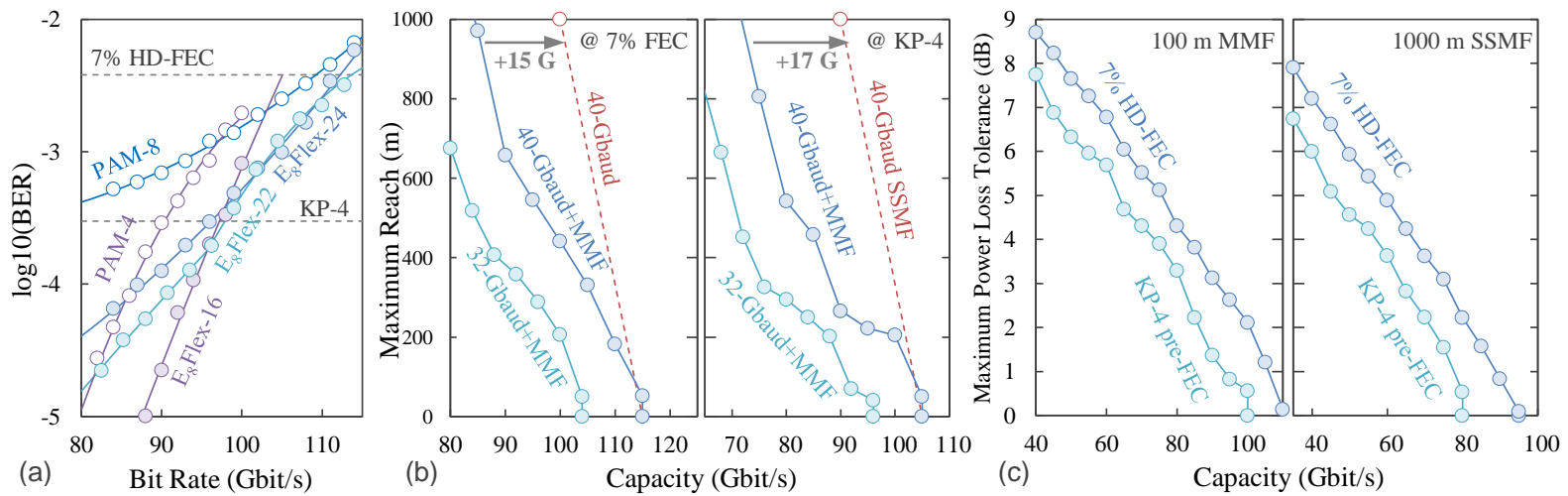

Fig. 3: (a) BER performance vs. bit rate in OBTB mode for conventional PAM and E8Flex formats. (b) Maximum reach vs. channel capacity for 7\% overhead hard-decision FEC BER threshold (left), and KP4 FEC BER threshold (right). (c) Maximum power loss tolerance vs. channel capacity for $100 \mathrm{~m}$ OM4 MMF link (left) and $1 \mathrm{~km}$ SSMF link (right).

pigtailed SM-VCSEL significantly outperforms MMF-pigtailed one at the fiber lengths from $200 \mathrm{~m}$. Meanwhile, SMF-pigtailed SM-VCSEL provides a possibility of transmission over standard ITU-T G.652 compliant SMF (SSMF). Thus, for further investigation on the features of the proposed transceiver scheme the SMFpigtailed SM-VCSEL is used.

Comparing with PAM signals: we observe from Fig. 3(a) that using both PAM-4 and PAM-8 at any symbol rate $100 \mathrm{G}$ net transmission is not achievable, but it can be achieved by 40Gbaud $E_{8}$ Flex- $n$, using fractional numbers of bit/sym. Noteworthy, $E_{8}$ Flex-16 and $E_{8}$ Flex-24 formats, which have the same bit/sym as to PAM-4 and PAM-8 respectively, outperform their conventional PAM counterparts due to the optimized geometrical features ${ }^{7}$.

Reach: Fig. 3(b) shows the maximum reach vs. the bit rate under the BER limits of different FEC schemes. The maximum reach values are estimated by fitting the results of transmission over 50, 100, 200, $500 \mathrm{~m}$ and $1 \mathrm{~km}$ MMF links. It is noteworthy that SSMF provides a gross $100 \mathrm{G}$ bit rate over $1 \mathrm{~km}$ link and outperforms the $500 \mathrm{~m}$ MMF link by the capacity $\times$ distance product.

Power penalty is estimated in Fig. 3(c) for different data rates over $100 \mathrm{~m} \mathrm{MMF}$ and $1 \mathrm{~km}$ SSMF links respectively. The general linearity of power penalty (SNR) vs. capacity (bit/s-sym) curves offers convenience in designing the capacity aware transceivers.

\section{Conclusions}

We propose a new flexible and fast 8D modulation family and experimentally verify its performance in 850nm SM-VCSEL based optical transceiver scheme. The proven merits of $\mathrm{E}_{8}$ Flex- $n$ include: (1) a smooth and swift software defined transition between bit rates without altering the hardware configuration or resampling; (2) higher sensitivity in the low noise regime; (3) optimized usage of hardware resources; (4) easy (de)mapping and (de)modulation procedures, utilizing PAM- $m$ signal as a basis; (5) intrinsic compatibility with the future industry standard of multi-lane optical interconnects, e.g. octal small-factor pluggable (OSFP). A single format in $E_{8}$ Flex- $n$ family can serve as a format for a specific fixed bit rate system, offering an optimum between the bandwidth utilization and system performance. Besides the IM-DD scenario, $\mathrm{E}_{8}$ Flex- $n$ can be also considered as a candidate for format and bit rate flexible transmission in coherent detection systems, too.

\section{Acknowledgements}

This work has been partly founded by Innovation Fund Denmark under the HOT project and the European Commission under the FP7 grant no. 619197 ADDAPT "Adaptive Data and Power Aware Transceivers for Optical Communications". The laboratory equipment was provided through the Polish Innovative Economy Program POIG.02.01.00-14-197/09 FOTEH project.

\section{References}

[1] M. Karlsson et al., "Spectrally Efficient Four-Dimensional Modulation," Proc. OFC, OTu2C.1, Los Angeles (2012).

[2] H. Bülow et al., "Polarization QAM modulation (POLQAM) for coherent detection schemes," Proc. OFC, OWG2, San Diego (2009).

[3] J. K. Fischer et al., "Bandwidth-Variable Transceivers based on Four-Dimensional Modulation Formats," J. Lightwave Technol., Vol. 32, no. 16, p. 2886 (2014).

[4] J. Renaudier et al., "Multi Rate IMDD Transceivers for Optical Interconnects Using Coded Modulation," Proc. OFC, Tu2J.2, Los Angeles (2015).

[5] M. Nakamura et al., "120-GBaud Coded 8 Dimensional 16 QAM WDM Transmission Using Low-Complexity Iterative Decoding Based on Bit-Wise Log Likelihood Ratio," Proc. OFC, W4A.3, Los Angeles (2017).

[6] H. Zhang et al., "Power-efficient $100 \mathrm{~Gb} / \mathrm{s}$ transmission over transoceanic distance using 8-dimensional coded modulation," Proc. ECOC, Th.2.2.1, Valencia (2015).

[7] X. Lu et al., "Optimized Eight-Dimensional Lattice Modulation Format for IM-DD 56 Gbit/s Optical Interconnections Using $850 \mathrm{~nm}$ VCSELs," J. Lightwave Technol., Vol. 35, no. 8, p. 1407 (2017). 